\title{
Le rôle des particules additives dans la construction de la cohésion discursive en langue maternelle et en langue étrangère
} MME Sandra Benazzo, MME Christine Dimroth, M. Clive Perdue, MME Watorek Marzena

\begin{abstract}
Sandra Benazzo, Christine Dimroth, Clive Perdue \& Marzena Watorek : Le rôle des particules additives dans la construction de la cohésion discursive en langue maternelle et en langue étrangère.

We compare the use of additive particles such as aussi ('also'), encore ('again, still'), and their 'translation equivalents', in a narrative task based on a series of pictures performed by groups of children aged 4 years, 7 years and 10 years using their first language (LI French, German, Polish), and by adult Polish and German learners of French as a second language (L2). From the cross-sectional analysis we propose developmental patterns which show remarkable similarities for all types of learner, but which stem from different determining factors. For the children, the patterns can best be explained by the development of their capacity to use available items in appropriate discourse contexts; for the adults, the limitations of their linguistic repertoire at different levels of achievement determines the possibility of incorporating these items into their utterance structure. Finally, we discuss to what extent these general tendencies are influenced by the specificities of the different languages used.
\end{abstract}

\section{Citer ce document / Cite this document :}

Benazzo Sandra, Dimroth Christine, Perdue Clive, Marzena Watorek. Le rôle des particules additives dans la construction de la cohésion discursive en langue maternelle et en langue étrangère. In: Langages, $38^{\mathrm{e}}$ année, n¹55, 2004. Construction du discours par des enfants et des apprenants adultes. pp. 76-105;

doi : https://doi.org/10.3406/lgge.2004.957

https://www.persee.fr/doc/lgge_0458-726x_2004_num_38_155_957

Fichier pdf généré le 03/05/2018 
Sandra Benazzo ${ }^{1}$

UMR 8528 (CNRS \& Lille 3)

\section{Christine Dimroth}

Max-Planck-Institut fuer Psycholinguistik, Nijmegen

Clive Perdue

UMR 7023 (CNRS \& Paris 8)

Marzena Watorek

UMR 7023 (CNRS \& Paris 8)

\section{Le rôle des particules additives dans la construction de la cohésion discursive en langue maternelle et en langue étrangère}

\section{O. INTRODUCTION}

Dans un contexte narratif, des items comme aussi, encore, de nouveau et toujours peuvent marquer respectivement l'addition d'entités (cf. 1) et l'addition d'intervalles temporels ( $c f .2-3$ ) pour une même situation.

(1) M. Bleu s'en va // puis M. Rouge aussi s'en va

(2) il dort // il dort toujours/encore

(3) il dort // il se réveille // puis il dort encore/de nouveau

L'acquisition de ces items, dorénavant "particules additives", constitue une tâche complexe en langue maternelle (L1) aussi bien qu'en langue étrangère (L2). Les langues diffèrent (a) par rapport au répertoire disponible de particules et par le type de relations additives encodées par chaque item, et (b) par rapport aux moyens utilisés pour le marquage de leur portée (position, accent, etc.). Nous avons analysé et comparé le rôle de ces particules dans le marquage des relations entre personnages et événements dans des textes narratifs initiés par une série d'images, produits par deux types d'apprenants : enfants francophones de 4, 7 et 10 ans (et, comparativement, des enfants germanophones et polonophones des mêmes âges), et apprenants adultes polonophones et germanophones du français de différents niveaux de compétence en L2. Ces productions ont été comparées à celles d'un groupe de contrôle composé d'adultes s'exprimant soit en français, soit en allemand, soit en polonais L1.

1. Adresses des auteurs: Benayzo, sandrabendzo@univ-lille3.fr,

Dimroth, christine.dimroth@mpi.nl, Watorek, Mar/ena.Watorek@univ-paris8.fr.

Nous remercions Christiane Marchello-Nizia et Claude Muller pour leurs remargues pertinentes. 
Pour les besoins des analyses présentées ici, nous adoptons une définition minimale du texte narratif : un événement complexe impliquant des protagonistes centraux est divisé par le locuteur en sous-événements occupant chacun un intervalle temporel borné et se suivant dans un ordre non-aléatoire - chronologiquement, en l'occurrence (on verra plus bas que la procédure de recueil de données adoptée impose une chronologie au locuteur). Les domaines référentiels centraux impliqués dans la construction d'une narration sont donc le temps et les entités agissantes. L'intégration des particules additives étudiées dans le discours dépend de la manière dont la référence aux entités et au temps est introduite, maintenue ou changée tout au long de la narration : les particules additives renforcent des chaînes anaphoriques dans ces domaines et jouent ainsi un rôle important en tant que moyens exprimant la cohésion discursive.

Les items visés par l'analyse apparaissent relativement tôt aussi bien en L1 qu'en L2: ils sont attestés en production spontanée bien avant l'âge des plus jeunes enfants considérés ici (cf. Gayraud 2004 pour le français, et Nederstigt 2004 pour l'allemand) et font partie du répertoire d'apprenants débutants en L2 (cf. Perdue, Benazzo et Giuliano 2002 pour le français L.2 ; Becker et Dietrich 1996, Dimroth 1998 et 2002, pour l'allemand L.2). La précocité dans l'emploi des particules additives est confirmée dans notre étude, mais la comparaison des deux types de populations (I.1 \& I.2) met en évidence, comme on le verra dans cette contribution, la différence de nature de leur tâche acquisitionnelle. Pour l'enfant, il s'agit d'apprendre à construire un discours cohérent et à marquer cette cohérence - la maîtrise progressive de ces items va donc de pair avec la maîtrise globale des relations anaphoriques entre entités discursives - tandis que pour l'adulte l'évolution concerne l'appropriation des moyens linguistiques spécifiques à la I.C.

la comparaison interlinguistique va nous permettre de distinguer les aspects évolutifs communs, liés à l'âge des enfants, des traits spécifiques aux I.C. Certains de ces traits seront précisés en 2 infra.

\section{LA TÂCHE ADDITIVE : RÉFÉRENCE AUX ENTITÉS ET AU TEMPS}

Dans cette tâche, le sujet doit produire une narration en ligne basée sur un ensemble de 30 images. L'intervieweur commente les quatre premieres images qui introduisent le cadre de l'histoire (un petit village), les personnages (monsieur bleu et monsieur rouge: $M B$ \& MR) et le but du récit (aider le roi à découvrir qui a délivré la princesse emprisonnée dans le château). Lee cadre spatio-temporel étant ainsi défini, le sujet doit construire son récit en précisant pour chacune des images suivantes ce qu'ont fait les personnages le jour où la princesse a été libérée.

Cette tâche est une adaptation de l'histoire additive conçue par Ch. Dimroth pour solliciter l'emploi d'items additifs tels que anch, noch, etc. 
(Dimroth 2002). Le tableau 1 résume brièvement le contenu de chaque image et précise les contextes favorables à l'emploi des items en question (épisodes additifs numérotés de I - IV). Les épisodes additifs sont organisés de la manière suivante:

Épisodes I et IV : concernent une série d'actions (partir, acheter une échelle, etc.) accomplies d'abord par l'un des personnages et plus tard par l'autre. La répétition des mêmes actions par des personnages différents devrait éliciter l'item additif aussi (p. ex. MR achète une échelle... MB aussi achite luc íchelle), ou en alternative, des expressions contextuellement équivalentes telles que également, comme $X$. Ces expressions marquent explicitement une relation d'addition entre les protagonistes pour un même type d'action.

Épisodes II-III : on voit dans des images consécutives le même personnage, Monsieur Rouge, qui dort (images 16 et 17), qui se réveille et lit un journal (image 18), et qui se remet à dormir (image 19). Cette série d'images demande des items marquant une relation additive dans le domaine temporel, puisque la même activité continue d'abord d'une image à l'autre (continuation $=$ encore/toujours), pour ensuite être répétée (réitération $=$ encore/de nouveau).

En alternative, on peut aussi interpréter ces images par rapport à l'horloge affichée : à 15 h 30 MR dort, à 16 h il dort, à 16 h 30 il lit un journal, à 17 h il dort.

\section{Tableau 1: La série d'images de l'histoire additive}

\begin{tabular}{|c|c|c|c|}
\hline $\begin{array}{l}\text { Épisode } \\
\text { Additif }\end{array}$ & $N^{\circ}$ image & Situation & Item additif \\
\hline & 01 & introduction " rue du village " & \\
\hline & 02 & introduction "princesse" & \\
\hline & 03 & introduction "Monsieur Rouge" & \\
\hline & 04 & introduction "Monsieur Bleu " & \\
\hline & 05 & $\mathrm{R} \& \mathrm{~B}$ sont devant l'église & \\
\hline \multirow[t]{10}{*}{$\mathbf{I}$} & 06 & $\begin{array}{l}\text { B part } \\
\mathbf{R} \text { reste devant l'église }\end{array}$ & \\
\hline & 07 & $\mathbf{R}$ part & aussi \\
\hline & 08 & $\mathbf{R}$ boit un jus d'orange au café & \\
\hline & 09 & $\mathbf{R}$ boit un $2^{\mathrm{e}}$ jus d'orange au café & \\
\hline & 10 & $\mathbf{R}$ boit un $3^{e}$ jus d'orange au café & \\
\hline & 11 & $\mathbf{R}$ part & \\
\hline & 12 & B marche dans la rue & \\
\hline & 13 & B fume une cigarette & \\
\hline & 14 & B part en bus & \\
\hline & 15 & $\mathbf{R}$ est assis sur un banc & \\
\hline
\end{tabular}




\begin{tabular}{|c|c|c|c|}
\hline $\begin{array}{l}\text { Épisode } \\
\text { Additif }\end{array}$ & $\mathbf{N}^{\circ}$ image & Situation & Item additif \\
\hline \multirow[t]{3}{*}{ II } & 16 & R dort sur le banc & \\
\hline & 17 & R dort sur le banc & encore/toujours \\
\hline & 18 & $\mathbf{R}$ est assis sur le banc et lit un journal 16.30 & \\
\hline \multirow[t]{6}{*}{ III } & 19 & R dort sur le banc & encore/de nouveau \\
\hline & 20 & $\mathbf{R}$ rentre dans un magasin & \\
\hline & 21 & $\mathbf{R}$ sort du magasin avec une échelle & \\
\hline & 22 & $\mathbf{R}$ marche vers la colline & \\
\hline & 23 & B arrive en bus & \\
\hline & 24 & B marche vers le café & \\
\hline \multirow[t]{6}{*}{ IV } & 25 & B boit un jus d'orange & aussi \\
\hline & 26 & B rentre dans le magasin & aussi \\
\hline & 27 & B sort du magasin avec une échelle & aussi \\
\hline & 28 & B marche vers la colline & aussi \\
\hline & 29 & $\begin{array}{l}\text { B monte la colline vers le château } \\
\mathbf{R} \text { reste à côté d'un arbre }\end{array}$ & \\
\hline & 30 & $\begin{array}{l}\text { B s'approche du château } \\
\text { R cueille des pommes }\end{array}$ & \\
\hline
\end{tabular}

Le marquage explicite des relations additives n'est jamais vraiment obligatoire.

Néanmoins, l'observation des productions des groupes de contrôle (natifs adultes francophones, germanophones et polonophones) révele que:

- l'addition d'entités de l'épisode IV est explicitement marquéc (tous moyens confondus: particules ou d'autres expressions) par $80 \%$ des sujets adultes français, allemands et polonais. L'épisode I par contre ne constitue pas toujours un contexte favorable pour ces relations : les pourcentages de sujets marquant cette relation se situent entre 20 et $30 \%$ dans les 3 langues. En effet, la séquence d'images correspondante permet l'expression d'un autre type de contraste, puisque les deux personnages sortent successivement de la scène mais dans deux directions opposées;

- pour l'addition d'intervalles temporels (épisodes II et III), entre 90\% et $100 \%$ des sujets français et allemands marquent respectivement la réitération et la continuité (tous moyens confondus : particules, préfixes verbaux, indication horaire ou quantification de la durée). Un pourcentage similaire est constaté en polonais pour la réitération (90\%) alors que le pourcentage est plus bas $(70 \%$ ) en ce qui concerne la continuité (cf. infra, exemple 27 ).

Par ailleurs, ces particules sont employées pour marquer d'autres relations additives possibles dans ce récit, en dehors des contextes I à IV. Nous pouvons donc en conclure qu'elles constituent pour les locuteurs natifs un 
moyen puissant de marquage de la cohésion textuelle, et nous avons choisi de quantifier dans les textes des apprenants l'ensemble des occurrences de ces particules (cf. section 2).

\section{LANGUES ET SUJETS}

\subsection{Les langues}

L'acquisition de ces particules en français L1 et L2 sera comparée avec l'acquisition d'items équivalents en allemand et polonais L1. Ces deux langues ont été choisies en ce qu'elles divergent par rapport au français à la fois dans le répertoire disponible pour encoder les relations additives et dans leur propriétés distributionnelles.

C'est König (1991) qui a proposé d'inclure les particules auch/noch et leurs 'équivalents' dans d'autres langues (aussi/encore, ...) dans la catégorie des particules additives. Dans un énoncé, une telle particule s'associe (Krifka 1998, cf. aussi la notion de "relate to » chez Reis et Rosengren, 1997), en fonction à la fois de sa position et du contexte discursif, à un constituant de l'énoncé et inclut ce constituant dans une gamme déjà établie par le contexte discursif. Pour reprendre l'exemple (1):

(1) M. Bleu s'en va// puis M. Rouge aussi s'en va. aussi s'associe à $M$. Rouge, rajoutant ainsi explicitement le personnage référé à la gamme des personnages partis. On peut comparer (1) à $\left(1^{\prime}\right)$ :

(1') M. Bleu s'en va// Il dit 'au revoir' aussi.

dans lequel aussi ajoute explicitement l'action de dire 'au revoir' aux actions accomplies par $M$. Bleu. On appellera le constituant auquel s'associe une particule additive son domaine d'application (DDA).

Ici n'est pas le lieu de faire une analyse approfondie de la sémantique de ces particules. Nous nous contenterons de quelques remarques pertinentes pour l'analyse des textes narratifs de notre corpus, ce qui nous amène à faire deux remarques préliminaires. Premièrement, nous adoptons l'heuristique des "équivalences de traduction" (aussi $=a u c h=t e z$, etc.), ce qui ne reflète certainement pas la complexité des relations entre systèmes. Nous sommes d'ailleurs conduits à analyser d'autres moyens linguistiques spécifiques à chaque langue, qui expriment en contexte les mêmes valeurs que les particules. Deuxièmement, s'agissant de productions d'apprenants, il est parfois difficile, même par une analyse contextuelle fine, de capter les valeurs d'une particule et nous signalons ces cas douteux aux endroits appropriés.

P'our l'addition d'entités discrètes, aussi peut s'associer librement (relativement à encore et toujours) à différents constituants de l'énoncé : à des entités et à des procès, comme dans (1), (1'), mais aussi à des lieux, des temps et des modalités. On parlera dans le cas d'aussi d'addition "non-restreinte". 
Encore s'associe aux entités, au temps et aux procès, mais avec une restriction : l'occurrence à laquelle cncore s'associe dans l'énoncé doit être du même type que l'occurrence précédente. Ainsi, " encore une bière " ajoute une occurrence de bière à une occurrence référée dans le texte précédent (encorél); "Jean a encore bu " ajoute le résultat d'une activité de beuverie à celui d'une autre activité de beuverie référée dans le texte précédent (encore 2 = réitération, fonction exprimée en français également par de nouveau). Une troisième utilisation d'encore est encore (!) plus restreinte, qui contraste deux points de référence temporelle exprimant la validité d'un procès à partir d'un temps antérieur et jusqu'au moment en question (encore' 3 = continuation). Cette utilisation rapproche encore d'une des significations de l'adverbe toujours, exprimant le prolongement de la validité d'un procès jusqu'au moment en question, ce qui est illustré dans l'exemple 2 supra.

Les particules centrales analysées dans les textes allemands sont auch ('aussi'), (immer) noch ('encore/toujours') et (schon) wieder ('de nouveau'). Dans les données polonaises, nous analysons les particules tez ('aussi'), je'szcze' (couvrant les trois sens de 'encore'), zhoz'u ("de nouveau') et ciagle/madal ('toujours"). Ces traductions sont approximatives car il n'y a pas de relation bi-univoque entre les termes français, allemands et polonais. La distribution forme-fonction entre les trois systèmes est approximativement ${ }^{2}$ la suivante:

Tableau 2 : Les particules centrales des trois langues

\begin{tabular}{|c|c|c|c|c|}
\hline Langue & $\begin{array}{l}\text { Addition non-res- } \\
\text { treinte }\end{array}$ & \multicolumn{3}{|c|}{ Addition restreinte } \\
\hline & $\begin{array}{l}\text { entités / lieu / } \\
\text { temps / procès }\end{array}$ & entités & \multicolumn{2}{|c|}{ intervalles temporels } \\
\hline & & & procès discrets & procès continus \\
\hline Français & aussi & encore $_{1}$ & $\begin{array}{l}\text { encore }_{2} \text { (une fois) } \\
\text { de nouveau }\end{array}$ & $\begin{array}{l}\text { encore }_{3} \\
\text { toujours }\end{array}$ \\
\hline Allemand & auch & noch & $\begin{array}{l}\text { (schon) wieder } \\
\text { nochmal }\end{array}$ & (immer) noch \\
\hline Polonais & tez & jeszcze $_{1}$ & $\begin{array}{l}\text { jeszcze }_{2} \text { raz } \\
\text { znowu }\end{array}$ & $\begin{array}{l}\text { jeszcze }_{2} \\
\text { nadal, ciagle }\end{array}$ \\
\hline
\end{tabular}

D'après le tableau, on peut remarquer que les trois langues disposent d'un item central spécifique pour l'addition non restreinte, alors qu'elles diffèrent pour l'encodage des relations temporelles : en français encore est plurifonctionnel, pouvant marquer aussi bien la réitération que la continuité, alors qu'en allemand et en polonais chacune des deux configurations temporelles

2. Chacune de ces particules peut avoir également d'autres valeurs (citons à titre d'exemple la valeur modale de auch ainsi que la valeur restitutive de aideder) que nous n'allons pas considérer ici. Par ailleurs le tableau ne rend évidemment pas compte des autres moyens d'expression de ces fonctions, qui sont commentés plus loin dans le texte. 
demande un item spécifique. Ces deux langues disposent également d'un item polyvalent, mais le sens réitératif n'est possible qu'avec une quantification temporelle explicite (jeszcze ${ }_{2} \mathrm{raz} /$ nochmal = encore une fois).

Les particules centrales pour l'addition non restreinte - aussi, auch et tezprésentent chacune une distribution spécifique. Pour le DDA le plus fréquent dans cette tâche, soit MB ou MR, sujet de l'énoncé, aussi et tez peuvent potentiellement occuper plusieurs positions syntaxiques, à savoir entre le SN sujet et le verbe, après le groupe verbal ou en fin d'énoncé. Suivant la position adoptée, aussi / tez se trouvent en position adjacente ou éloignée par rapport à leur DDA ( $\underline{M B}$ aussi achète une échelle vs $\underline{M B}$ achète aussi une échelle vs $\underline{M B}$ achète une échelle aussi).

Auch a par contre une position fixe : il est généralement placé dans le Mittelfeld, soit entre le verbe fléchi et le verbe non-fléchi ${ }^{3}$ :

(1") H. Rot ist auch weggegangen.

MR est aussi parti

Son DDA, qui n'est souvent pas identifiable par la seule position, est alors signalé par une courbe intonative particulière (la "Brückenakzent »).

La distribution des particules à valeur temporelle est plus homogène: encore/toujours ainsi que (immer) noch/wieder se trouvent généralement placées après le verbe fléchi; la particule polonaise de la réitération znowvu se place davantage avant le verbe, tandis que les items marquant la continuité nadal/wciaz/jeszcze peuvent occuper aussi bien la position pré- que postverbale sans que l'on note un changement de sens.

À partir de cette (trop) brève description, on peut formuler différentes hypothèses sur le développement de ces moyens chez l'apprenant. On s'attend à ce que le jeune enfant commence à utiliser une particule additive généralisée, éventuellement idiosyncrasique ${ }^{4}$, sans restrictions sémantiques. Pour l'addition restreinte ( $c f$. tableau 2), la répétition, qui implique l'association de la particule à une entité de même type, ou bien à un seul intervalle temporel, devrait précéder la continuation qui nécessite la prise en compte de deux repères temporels 5 . La séquence :

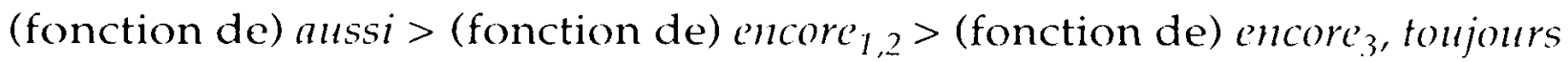

\footnotetext{
3. D'autres positions, en principe possibles en allemand (par exemple la position initiale), ne sont pratiquement pas attestées dans lees données ici analysées (cf. note 8 ).

4. L'enfant anglophone se forge parfois une particule portmanteau "moreagain " [mo:gen] (Benjamin l'erdue, comm. personnelle).

5. Dans le domaine de l'espace, Johnson \& Slobin (1979) ont constaté l'acquisition tardive a travers les langues de la préposition entre (et équivalents de traduction), qu'ils expliquent par la nécessaire prise en compte de deux reperes spatiaux. Comme on le verra par la suite, certains locuteurs contrastent les deux persomnages MR et MB en marquant la simultanéité des actions qu'ils accomplissent. ()r, exprimer la simultanéité implicyee de situer deux actions distinctes par rapport a un seul moment repere et on s'attend donc a ce que la simultanéité soit aussi exprimée tardivement par les apprenants enfants.
} 
ne devrait pas être pertinente pour l'apprenant adulte (et cognitivement développé). Pour lui se pose un problème de distribution : où placer la particule pour que son DDA soit identifiable? Et (autre chose) où placer la particule pour être conforme à la distribution de la I_C ? Ces questions valent évidemment pour l'apprenant de L1 aussi. Notre analyse contrastive nous permet de faire remarquer que le placement moins variable des particules en allemand devrait en faciliter l'acquisition pour cette langue.

Faisons une dernière remarque à propos d'autres moyens possibles pour exprimer les fonctions ci-dessus. Le français semble employer des moyens expressifs plus variés que l'allemand. Pour la répétition, signalons le préfixe $r c^{\prime}-V$ dont la distribution est bien plus régulière que celle des particules et qui pourrait donc être acquis relativement tôt. Tous les moyens mentionnés jusqu'ici s'accommodent, dans un texte narratif, de l'ordre chronologique des événements: il n'est pas à exclure que d'autres moyens encore viennent à être utilisés dès lors que le répertoire de l'apprenant lui permet de sortir de cette contrainte discursive, en exprimant la simultanéité, par exemple (cf. note 5).

\subsection{Les sujets}

Les tableaux suivants donnent le total des sujets sur lesquels portent nos analyses. Pour les trois langues, les enfants sont des écoliers qui ont été interviewés dans leur école. Les adultes, apprenants et natifs, sont de niveaux socio-économiques variés. Nous renvoyons à la présentation de ce numéro pour de plus amples détails.

Tableau 3 : Sujets s'exprimant en L1

\begin{tabular}{|l|l|l|l|}
\hline \multirow{4}{*}{ Français } & groupe 4 ans & 15 sujets & tot. 45 \\
\cline { 2 - 4 } & groupe 7 ans & 15 sujets & \\
\hline & groupe 10 ans & 15 sujets & \\
\hline \multirow{4}{*}{ Allemand } & groupe 4 ans & 10 sujets & tot. 27 \\
\hline & groupe 7 ans & 8 sujets & \\
\hline & groupe 10 & 9 sujets & tot. 30 \\
\hline \multirow{4}{*}{ Polonais } & groupe 4 ans & 10 sujets & \\
\hline & groupe 7 ans & 10 sujets & \\
\hline & groupe 10 ans & 10 sujets & \\
\hline
\end{tabular}

Tableau 4 : Sujets s'exprimant en L2

\begin{tabular}{|c|c|c|c|c|}
\hline $\begin{array}{l}\text { Groupe } 1 \\
\text { déb. et interm. }\end{array}$ & $\begin{array}{l}10 \text { adultes polonophones } \\
\text { (dont } 2 \text { déb. et } 8 \text { intermé- } \\
\text { diaires) }\end{array}$ & $\begin{array}{l}\text { de Bac } \\
\text { à Bac }+4\end{array}$ & $\begin{array}{l}\text { arrivés en France depuis } \\
1 \text { à } 6 \text { mois } \\
\text { (+ } 1 \text { sujet depuis } 2 \text { ans) }\end{array}$ & $\begin{array}{l}\text { âgés de } 20 \\
\text { à } 46 \text { ans }\end{array}$ \\
\hline $\begin{array}{l}\text { Groupe } 2 \\
\text { avancés }\end{array}$ & $\begin{array}{l}10 \text { adultes } \\
\text { polonophones }\end{array}$ & $\mathrm{Bac}+1$ & $\begin{array}{l}1^{\text {re }} \text { année universitaire à } \\
\text { Lublin }\end{array}$ & $\begin{array}{l}\text { âgés de } 19 \\
\text { à } 21 \text { an }\end{array}$ \\
\hline $\begin{array}{l}\text { Groupe } 3 \\
\text { avancés }\end{array}$ & $\begin{array}{l}10 \text { adultes } \\
\text { germanophones }\end{array}$ & $\begin{array}{l}\text { de Bac }+1 \\
\text { à Bac }+5\end{array}$ & $\begin{array}{l}\text { arrivés en France depuis } \\
1 \text { à } 6 \text { mois }\end{array}$ & $\begin{array}{l}\text { âgés de } 21 \\
\text { à } 30 \text { ans }\end{array}$ \\
\hline
\end{tabular}




\subsection{Le corpus}

Le tableau suivant donne le nombre de particules relevées par groupe de sujets (il faut garder à l'esprit que les groupes sont quantitativement non homogènes). Ces chiffres ne peuvent cependant donner qu'une idée approximative de l'expression des relations additives étant donné que des moyens alternatifs d'expression des mêmes relations (par exemple, fr. re-, de nouveau ou également) ainsi que certaines combinaisons complexes de ces particules (surtout en allemand) y sont absents :

\section{Tableau 5: Total des occurrences}

\begin{tabular}{|l|c|c|c|c|}
\hline & Français L1 & Français L2 & Allemand L1 & Polonais L1 \\
\hline aussi & 41 & 73 & & \\
\hline encore & 56 & 40 & & \\
\hline toujours & 71 & 6 & & \\
\hline \hline auch & & & 109 & \\
\hline (immer) noch & & & 78 & \\
\hline (schon) wieder & & & 140 & \\
\hline \hline tez & & & & 20 \\
\hline jeszcze & & & & 22 \\
\hline nadal & & & & 33 \\
\hline znow & & & & \\
\hline
\end{tabular}

\section{RÉSULTATS EN L1}

Les résultats de l'analyse seront exposés suivant le type d'addition (entités is intervalles temporels) pour les trois tranches d'âge. Nous allons centrer l'attention sur le développement constaté en français L1, en signalant au fur et à mesure si, à parité d'âge, l'évolution en allemand et en polonais est similaire et/ou pour quels aspects elle s'en démarque.

\subsection{Tendances évolutives pour l'addition d'entités}

En ce qui concerne le français L1, pour les 3 tranches d'âge analysées, l'addition d'entités est essentiellement marquée à l'aide de aussi. L'évolution dans les emplois de cette particule dans la tâche additive est étroitement liée au développement concernant la référence aux entités.

\subsubsection{Les enfants de 4 ans}

Les occurrences de alssi à 4 ans (12 au total) se démarquent des occurrences des enfants plus âgés pour deux raisons:

1) aussi s'associe à des entités extra-discursives (4 occ.)

2) l'identification du IDIDA de la particule s'avere difficile (8 occ.) 


\section{1) référence à des entités extra-discursives}

Aussi peut apparaître en association avec des déictiques spatiaux qui réfèrent directement à l'image support. Ce même phénomène est observé dans lés données en allemand et en polonais L1. En (4a, b, c), p. ex., l'enfant constate ainsi une similarité entre deux images consécutives :

(4a) là il est allongé // là aussi (Lucie)

(4b) da schläft er // da schläft er auch (Flo) là dort il // là dort il aussi

(4c) a tutaj spi // a tutaj tez spi (Wik) e't lì dort // èt là aussi dort

On peut pareillement parler d'un emploi déictique des pronoms personnels en $(5 \mathrm{a}, \mathrm{b})$ : dans les deux extraits, les énoncés en question font référence à deux images distinctes qui montrent (5a) d'abord le départ de monsieur bleu, et ensuite le départ de monsieur rouge, et en (5b) d'abord MR en train de cueillir des pommes et puis $\mathrm{MB}$ qui semble s'approcher d'un arbre. Comme l'enfant ne donne pas d'indices linguistiques pour le changement de la référence personnelle, le référent n'est pas identifiable:

(5a) le monsieur il s'en va / / il s'en va aussi (Tiphany)

(5b) der rote will die äpfel runterholen // und da will er auch die äpfel runterholen

a'er demln?

der blaue $(A n i)$

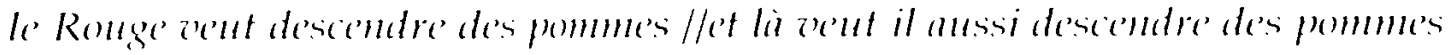
mais ipui?

le ble'll

On n'observe pas d'emplois déictiques des pronoms personnels en polonais, ce yui est évidemment dû à ses propriétés de langue à sujet nul. En revanche le référent correspondant au DDA de la particule additive peut s'avérer également difficilement identifiable, entre autrés parce que le changement de la référence aux entités agissantes n’est pas signalé.

(5) a tutaj niesie drabine

a tutaj idzie

a tutaj tez jed $z$ ic autobusem (Wik) et ici (s) porte échelle (=MR)

et ici $(x)$ marche (=MR)

et icianssi Ø z'a e'n bus (=MB)

les emplois de aussi, auch ét tez attestés dans ces énoncés sont cependant tout a fait en accord avec les caractéristiques de la production de cet âge dans les 3 langues, à savoir:

- l'ancrage déictique du récit: lés énoncés de l'enfant sont souvent des commentaires référant directement à l'image au lieu d'être reliés pour constituer un véritable récit.

- la faible distinction des contextes informationnels : en français et en allemand certains sujets utilisent un pronom ou SN aussi bien dans le cas de maintien que de changement de la référence aux protagonistes; en polonais c'est l'anaphore zéro qui peut figurer dans les 2 contextes informationnels. 
Ainsi, globalement $50 \%$ des sujets en français et en allemand, et $60 \%$ en polonais, produisent des énoncés a mbigus si bien que l'étayage de l'adulte devient nécessaire pour identifier les protagonistes/entités nommées (on voit cela dans les extraits suivants où les interventions de l'adulte sont données en italique).

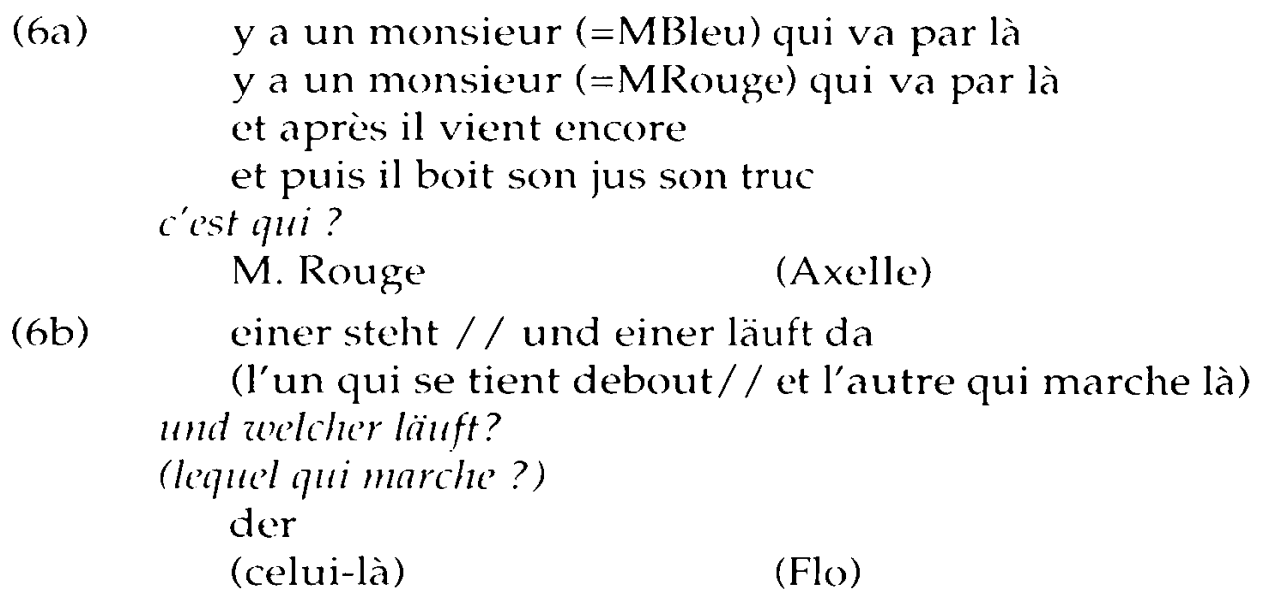

2) Interprétation variable du DDA (la position de aussi admet plusieurs interprétations du DDA)

Mis à part les occurrences où aussi est associé à des déictiques, son DDA peut s'avérer ambigu de par son positionnement syntaxique. Plusieurs positions sont attestées (en tête d'énoncé, en fin d'énoncé et entre le verbe et l'objet), qui admettent toutes différentes interprétations quant au DDA.

(7) après il va dans le magasin

(il) va acheter une échelle aussi (Mélissa)

Dans l'exemple (7), de par son positionnement alussi pourrait aussi bien porter sur le protagoniste (signifiant ainsi : $M B$ aussi achète une échelle comme $M R$ avant), que sur le prédicat "acheter une échelle » (= il va dans le magasin et en plus il achète une échelle), ou encore marquer un lien additif entre énonciations

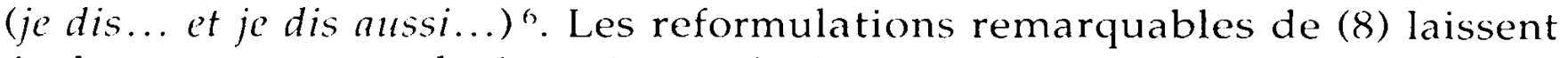
également ouvertes plusieurs interprétations.

(8) et il prenda aussi une échelle et $M$. Bleu monta aussi pour cueillir des... et aussi M. Bleu prena l'échelle... avait pris l'échelle pour aussi monter un arbre (Lena)

Ces difficultés d'interprétation liées à la mobilité de aussi semblent typiques du français. En effet, à cet âge, l'allemand auch est systématiquement placé dans le Mittelfeld, conformément à la langue cible. Le DDA peut se trouver avant ou après le verbe, l'intonation l'identifiant clairement.

(9a) der rote war auch weggelaufen (Vic) le rouge '́tait aussi parti

(9b) jetzt hat der auch eine leiter mitgenommen (Lou) maintenant a il aussi une échelle pris

6. Cedernier emploi a d'ailleurs été remarqué dans plusieurs études. 
Tez se trouve de préférence dans la position préverbale. Si le sujet qui constitue son DDA est laissé implicite, tez est en position initiale. Cependant, dans les contextes favorables au marquage d'addition d'entités, dans les données de l'allemand ainsi que du polonais, on retrouve souvent les particules réitératives wieder/znozen au lieu de alach/tez. I a situation de prendre une échelle (10a) et de se diriger vers le château (10b) est effectivement répétée dans l'histoire, mais elle est effectuée par deux personnages différents.

(10) und der lauft schon wieder mit die leiter (Jul)

et il marche de nowienta aie'c l'éche'lle'

(10b) PC zrywa jablka // PN znow idzie do zamku (Fil)

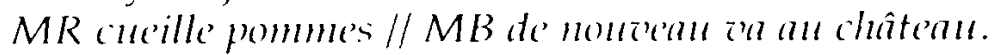

Ces emplois de zoieder/znozen manifestent la reconnaissance de la part de l'enfant qu'une partie de la situation en question se répète. Ils laissent cependant ouvertes deux interprétations : l'enfant pourrait marquer soit la réitération du procès (sans préciser que l'entité agissante changé), soit la similarité entre images (je vois une deuxième fois la même situation).

La " confusion " entre particules additives et réitératives n'est par contre pas attestée en français, sauf dans l'autre sens : emploi de là aussi pour la continuité (ex. 4). On y reviendra dans la section consacréc à l'addition d'intervalles temporels. A ce stade, on peut donc supposer que l'enfant ne maitrise pas encore les différences sémantiques entre différents types d'addition, ce qui va dans le sens de notre premiere hypothèse.

\subsubsection{Les enfants de 7 ans}

I es difficultés relevées a 4 ans pour l'interprétation du IDI)A de aussi sont dépassées dans la production des 7 ans. $A$ cet âge, l'addition d'entités est souvent exprimée par l'association de la particule au pronom contrastif $/ 11 i^{7}$ qui permet de signaler clairement, par reprise anaphorique, le constituant affecté (6 occ. sur 11):

(11) lui aussi a pris une échelle et il est allé lui aussi au château (Mélodie)

ou encore par le positionnement de la particule en position adjacente à son DDA, soit apres le SN sujet (3 occ. sur 11):

(12) M. Bleu i(I) part / / et M. Rouge aussi i(l) part (Matthias)

Ces emplois s'intégrent dans l'évolution concernant la référence aux entités qui est constatée dans les 3 langues. A cet âge, les protagonistes sont clairement identifiés, voire surexplicités : en français et en allemand l'emploi du pronom est réduit au maintien strict de la référence, alors que le SN plein peut être encore utilisé non seulement dans les contextes de changement de la référence

7. I e pronom lui est sporadiquement présent che\% les 4 ans, mais son emploi à cet age, certainement contrastif, ne permet pas d'identifier les personnages: "il (=MB) s'est arrété// lui (M. Jame) il conduit " (Tiph.). 
(le $M B$ il... le $M R$ il...), mais aussi dans les contextes de maintien, ce qui serait inapproprié dans la production adulte.

(13a) der herr rot geht da was trinken / / und der herr rot trinkt da wieder was (Lin)

le M. Rouge va là boire quelque chose // èt le M. Rouge boit là encore quelquechose

L'entité maintenue peut aussi recevoir un double marquage dans un même énoncé - par un pronom et un SN plein.

(13b) dann trinkt der erst mal was, der herr rot (Han)

puis boit il d'abord quelque chose le $M$. rouge

Comme c'était le cas à quatre ans, la particule tez est utilisée à 7 ans de préférence en position pré-verbale, soit avec le SN plein en tête d'énoncé (6 fois SN-tez-V) soit avec sujet implicite (6 fois Ø-tez-V), qui est désormais limité au maintien strict de la référence. Dans ce même contexte, on constate cependant, comme en français, la présence du SN plein ou bien d'un pronom.

(13c) PN tez biegl w kierunku zamku (Maciej) $M B$ aussi courait dans la direction du château

Cette relative redondance dans le marquage de l'information semble donc se traduire par la claire explicitation du DDA d'aussi, voire par l'établissement de comparaisons explicites entre les protagonistes qui effectuent les mêmes actions (ex. 14) à l'aide de comme en francais (et équivalents en allemand et en polonais) :

(14a) lui aussi il part // il prend une échelle aussi

il va vers le chemin comme son frère (Daphnis, unique occurrence de commene)

(14b) jetzt geht der auch dahin wie herr rot maintenant va il aussi là bas comme M. Rouge (Van)

(14c) potem poszedl do sklepu z drabinami après est allé au magasin avec lés échelles jak I'C kupil jedna drabine comme MR a acheté échelle' (Magdalena)

On voit donc à 7 ans deux types d'évolution par rapport aux apprenants plus jeunes: (a) les antécédents des particules se trouvent tous dans le co-texte, ce qui peut fournir une explication au phénomène de sur-marquage des liens anaphoriques (pour reprendre la métaphore de Slobin (1978), l'enfant de 7 ans met un "projecteur » sur cet aspect de la construction du discours); (b) les différentes valeurs de chaque particule semblent être maintenant maîtrisées.

\subsubsection{Les enfants de 10 ans}

Le processus de diversification de la référence aux éntités se poursuit à 10 ans. En français aussi bien qu'en allemand, le maintien de la référence peut être exprimé par un pronom ou bien par l'anaphore zéro (disparition du SN), et, dans ce contexte informationnel, l'anaphore zéro s'impose sur les pronoms en polonais. 
En français, les actions des protagonistes sont maintenant contrastées par plusieurs moyens : le pronom contrastif lui, les conjonctions tandis que/pendant que/alors que.

(15) M. Bleu lui monte vers le château...

tandis que MR a déjà cueilli des pommes (Lucie, 10 ans)

M. Rouge appuie son échelle contre un pommier...

// pendant que monsieur Bleu arrive. (Axelle, 10 ans)

La combinaison pronom contrastif + aussi devient plus mobile à l'intérieur de l'énoncé. Contrairement aux occurrences des 7 ans, cette particule est placée de préférence (7 occurrences sur 8) en position postverbale, accompagnée ou non par le pronom contrastif (cf. tableau 7.1 en annexe et exemples suivants).

(16) après il va voir vers le magasin d'outils / / et $\varnothing$ en sort lui aussi avec une échelle (Émeric, 10 ans)

il se dirige aussi vers le château. (Nicolas, 10 ans)

Aussi reste la particule privilégiée pour exprimer l'addition d'entités, mais la structure informationnelle du discours devient plus complexe. Dans l'extrait (18), Nicolas établit un cadre lui permettant d'exprimer la similarité entre les actions des deux protagonistes (fait la mêne' chose' comme $M R$ ), puis explicite les actions concernées :

(18) et il fait la même chose comme MR

il achète une échelle

il se dirige aussi vers te château (Nicolas, 10 ans)

Les occurrences de aussi à cet âge sont très similaires à celles qu'on retrouve chey les adultes natifs. I'évolution ultérieure concerne plutôt la diversification des moyens mis en (euvre par ces derniers (égaleme'nt, ì son tour, etc.), pour souligner que les protagonistes répètent les mêmes actions.

(19) il en ressort avec une échelle sur le dos et $\varnothing$ se dirige lui aussi à son tour vers le château (Bruno, adulte)

(20) il boit également une orangeade il va également acheter sa petite échelle (Linda, adulte)

Cette diversification des moyens qui caractérise la production des adultes en français est également constatée en polonais L.1, mais dans une bien moindre mesure en allemand L1 (cf. tableaux 6.1-6.3).

L'évolution dans l'emploi des particules additivés auch et téz dépend également de la diversification des moyens utilisés pour la référence aux entités, mais le résultat est différent : suite à l'augmentation du maintien de la référence par anaphore zéro, le DDA de ces particules est souvent implicite. Ce phénomène n'est attesté qu'une seule fois chez les adultes francophones:

(21) M. Bleu sort de la quincaillerie avec une échelle et $\varnothing$ monte aussi en direction du château (Nicolas, adulte) 
Auch reste toujours en position fixe dans le Mittelfeld", alors que tez semble devenir plus mobile, occupant également des positions postverbales: cette mobilité est liée à l'emploi d'un ordre des mots plus varié, puisque la particule accompagne presque toujours son DDA.

Une dernière différence remarquable au niveau interlinguistique concerne la quantité des particules additives : très nombreuses en allemand et relativement réduites en français à tous les âges. Nous retournerons à cette différence infra dans la discussion.

L'annexe 1 (tableaux 6 et 7) résume et quantifie l'évolution constatée dans les trois langues par rapport aux moyens utilisés pour marquer l'addition d'entités et par rapport à la position des particules additives dans des énoncés contenant un verbe.

\subsection{Tendances évolutives pour l'addition d'intervalles temporels}

Dans le domaine des procès, la tâche additive demande de marquer l'addition d'intervalles temporels discrets (réitération) et non discrets (continuation) et, facultativement, la simultanéité, quand les 2 protagonistes sont présents dans la même image.

\subsubsection{Les enfants de 4 ans}

Comme nous avons déjà remarqué plus haut, on retrouve dans les productions des 4 ans en polonais et en allemand, tout comme en français, des particules telles que aussi/tez/auch dans les contextes temporels en raison du fait que l'enfant se limite à constater la similarité entre images consécutives. Si on exclut ces occurrences, encore est la seule particule attestée dans les épisodes additifs de nature temporelle à 4 ans. Dans les contextes de réitération, cette particule est en compétition avec le préfixe verbal re $+\mathbf{V}$, avec la même proportion quantitative ( $5 \mathrm{re}-\mathrm{V}$ et 5 encore) :

(22) il dort // il lit son journal // il redort il dort // il lit une histoire // il dort encore

(Quentin, 4 ans.)

(Lucie, 4 ans)

alors que cette même particule est dominante dans les contextes de continuité. On peut cependant se demander si encore marque vraiment toujours la continuité temporelle du procès. Quelques doutes sont justifiés par des exemples tant en français qu'en allemand.

$$
\text { il s'allonge // il s'allonge encore }
$$$$
\text { (Lola, } 4 \text { ans) }
$$

En (23) la scène à décrire est l'épisode II : MR continue de dormir. La lecture continuative de encore est ici incompatible avec le type de procès sélectionné.

8. Chey les adultes, il y a seulement 2 occurrences de la particule en tête d'énoncé (" auch herr blau kauft sich eine leiter " (Ina) : atussi MB achite unle 'schelle, if. Tableau 7.2). 
Mais, en raison de la polyvalence de encore en français, il est difficile d'établir quelle est sa valeur dans ce contexte. Nous retrouvons la difficulté de ces enfants de 4 ans à distinguer, en contexte, les associations forme/valeur de la langue cible.

Si on regarde les données des enfants allemands de cet âge, on constate une présence massive de wiéder (l'équivalent de encore réitératif), qui est parfois utilisé pour les contextes d'addition d'entités (à la place de aussi) et de continuité (à la place de immer noch):

(24) (contexte de continuité)

HR schläft auf der bank

MR dort sur le banc

HR schlät wieder auf der bank (Joe)

MR dort de nourean sur le banc

La valeur de continuité est correctement marquée par lieģ immér noch par deux enfants, dont un seul qui arrive à opposer les deux relations temporelles comme en langue cible (continuation : immer noch, réitération: schon zoieder). Ces exemples étayent l'hypothèse que les enfants de cet âge utilisent parfois encorc/aviéder pour marquer simplement la répétition d'une situation déjà vué (cf. lì aussi), alors que la continuité pose problème, du moins dans cette tâche.

En polonais par contre, les sujets de cet âge n'utilisent pas du tout la particule potentiellement passe-partout jeszcze', qui marque tant la continuité que la réitération, mais les deux contextes temporels ne sont en général que très faiblement maryués.

\subsubsection{Les enfants de 7 et de 10 ans}

Ie développement ultérieur ché les enfants francophones de 7 et 10 ans est constitué d'une part par le marquage quantitativement croissant des deux contextes temporels, et d'autre part par la diversification progressive des moyens utilisés à cette fin : le préfixe verbal re- $\boldsymbol{V}$ devient largement majoritaire en cas de réitération (il se' remet à dormir ou bien il se rendort), alors que toujours émerge à 7 ans et s'impose à 10 ans pour marquer la continuité. Cette préférence pour toujours est encore plus nette chè les adultes, où encore' n'est plus attesté qu'une seule fois (jusqu'à 10 ans, on retrouve un ou deux sujets présentant un marquage erroné ou douteux dans ces deux contextes).

(25) il s'endort // il dort toujours // après il lit // il dort... (Matthias, 7 ans) il s'endort // il dort toujours // il s'est réveillé // il lit son journal / / puis il se rendort (Lucie, 10 ans)

Parallèlement, la simultanéité des actions des protagonistes par rapport à un même intervalle temporel commence à être signalée à partir de 7 ans.

(26) MR il cueillit des pommes / pendant ce temps MB il continue toujours à avancer (Nicolas, 10 ans) 
La production des enfants allemands et polonais, dans les tranches d'âge supérieures aux 4 ans, montre la spécialisation de l'emploi de chaque particule dans les contextes appropriés. La continuité est toutefois généralement peu marquée en polonais, cette valeur peut être en effet exprimée par l'opposition aspectuelle perfectif/imperfectif comme le montre l'exemple suivant :

$\varnothing$ zasnal na lawce // spi // zasnal znowo

(MR) s'est endormi // dort (imperfectif) // s'est endormi de nouveau

Par rapport aux enfants francophones, le marquage de la simultanéité semble être un peu plus tardif : en polonais, cette relation commence à être marquée à 10 ans seulement, alors qu'en allemand elle est très rare, et n'est attestée que chez les adultes.

L'annexe 2 (tableau 8) récapitule et quantifie l'évolution que nous venons d'esquisser, en détaillant les items constatés par tranche d'âge et par langue.

\subsection{Résumé $L 1$ : traits développementaux partagés par les enfants}

On peut décrire l'évolution des productions à travers les 3 tranches d'âge de la façon suivante.

À 4 ans, les enfants éprouvent des difficultés, face à l'exercice demandé, à en faire un récit.

Les productions de ces jeunes enfants tendent vers la description de chaque image individuellement, ce qui réduit fortement l'expression, d'énoncé en énoncé, de liens temporels ou de contrastes des personnages. Les liens additifs exprimés sont plutôt d'ordre extralinguistique (déictique) ou métadiscursif (description de nowveau d'une scène déjà décrite). Il en résulte une sur-généralisation de certaines particules à des contextes inappropriés en comparaison avec les productions adultes.

À 7 ans, l'attention des enfants semble dirigée vers la construction du discours.

Les liens additifs sont établis par rapport aux informations exprimées dans les énoncés précédents, les usages déictiques ou métadiscursifs ayant quasiment disparu. À cet âge, le répertoire de ces enfants leur permet de distinguer clairement l'addition des entités d'une part et des relations temporelles (réitération et continuité) d'autre part. On constate même une sur-utilisation de certains moyens linguistiques aussi bien pour le maintien de la référence (SN plein + pronom) que pour l'addition d'entités (combinaison de la structure lui aussi + comme $\mathrm{x}$ ), ce qui manifeste leur souci d'exprimer clairement la référence aux entités à partir du contexte discursif.

Chez les enfants de 10 ans, on observe le résultat d'un ajustement des moyens disponibles pour la référence aux entités, accompagné d'un emploi 
plus souple d'aussi (et équivalents). Ajustement également dans l'expression des liens temporels : pour le français, toujours s'emploie de manière privilégiée pour la continuité, et re- $V$ pour la réitération, cette dernière forme prenant le pas sur encore', comme l'atteste le tableau 8.1.

On voit émerger un moyen plus complexe pour mettre en relation les deux protagonistes, à savoir l'expression de la simultanéité. C'est donc à 10 ans que les enfants utilisent des moyens linguistiques permettant d'échapper au principe de l'ordre chronologique, comme l'attesté également leur emploi d'un cadre temporel portant sur une série d'actions.

\section{RÉSULTATS EN FRANÇAIS L2}

Rappelons que nous avons analysé la production de trois groupes d'apprenants du français, L,2 : un groupe de polonophones débutants et intermédiaires, un groupe de polonophones avancés, et un groupe de germanophones avancés.

\subsection{Addition d'entités}

\subsubsection{Le niveau Débutant - Intermédiaire}

Aussi est la particule la plus fréquente pour le marquage de cette relation, méme si d'autres moyens sont également présents. Contrairement aux enfants de 4 ans, l'adulte distingue clairement entre les contextes informationnels de maintien zes changement de la référence.

Che\% Waldemar, yui se situe à un niveau basique et est le seul apprenant à ne pas disposer de pronoms de 3" personne, on constate en cas de maintie'n, l'utilisation de l'anaphore zéro en alternance avec le SN.

(28) ici monsieur acheter échelle

et $\varnothing$ parti pour le château...

$M B$ venir en face à la château.

Les autres débutants de ce groupe se servent alternativement du pronom ou du SN plein pour le maintien. Dans leurs productions, le DDA de aussi est clairement identifiable, puisque la particule est souvent placée immédiatement après son DDA :

(29) M. Rouge juste à côté M. Bleu / / il aussi devant l'église

M. Bleu parti // M. Rouge aussi partir (Ewa)

Chez les intermédiaires elle se trouve, par contre, de préférence après le groupe verbal (30), ce qui, en l'absence du pronom contrastif lui, laisse ouvertes plusieurs interprétations sur le DDA.

(30) après MR aller parti aussi (Agn.) 
L'ambiguité potentielle de cette position syntaxique pourrait expliquer les reformulations fréquentes qu'on retrouve, comme dans l'extrait suivant :

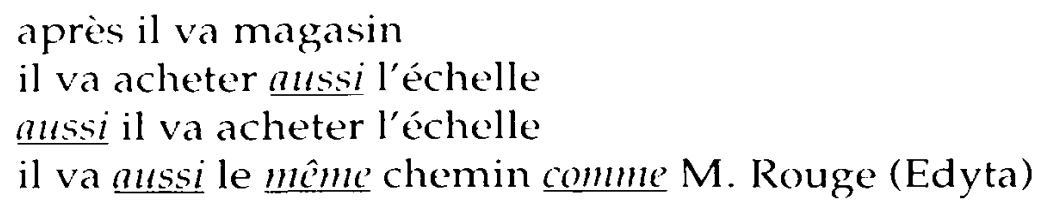

Dans les contextes favorables à l'emploi d'aussi, on retrouve également l'emploi d'autres expressions, listées sous (32), y compris l'emploi idiosyncrasique de à nouveau qui semble contextuellement correspond re à aussi:

(32) (a) M. Bleu il assis comme avant M. Rouge // et il boire quelque chose comme avant $M$. Rouge (Barb)

(b) il prend le même passage petit à château (Paw)

(c) M. Bleu à nouveau boit (Agn)

\subsubsection{Le niveau avancé}

Les productions des apprenants avancés ne laissent pas de doute quant à la référence aux entités : ces apprenants utilisent le SN plein pour le changement de la référence et la référence pronominale en cas de maintien. La diversification des contextes informationnels s'arrête pourtant là : l'anaphore zéro en cas de maintien est absente des productions des polonophones et rare chez les germanophones (attestée chez 3 sujets pour un total de 5 occurrences); aucun des sujets ne présente de procédés de mise en contraste des protagonistes constatés chez les adultes natifs (tandis que, alors que, etc.).

Pour l'addition d'entités, aussi est la particule la plus fréquente chez ces apprenants, bien que les deux groupes diffèrent nettement en termes quantitatifs : chez les germanophones, on relève 34 occurrences de la particule, soit le double de chez les polonophones (17 occurrences). Si on compare ces chiffres aux données de L1, l'influence de l'allemand sur la production L2 semble assez claire, car tant les francophones natifs que des autres groupes d'apprenants emploient aussi bien moins fréquemment.

Dans les deux groupes, aussi peut occuper plusieurs positions syntaxiques, mais le placement postverbal est nettement le plus fréquent, en particulier chez les germanophones (voir tableau 9); la différence principale intergroupe réside dans les autres positions attestées : bien que les nombres soient faibles, on voit que les polonophones montrent une légère préférence pour la position préverbale, alors que les germanophones utilisent davantage la position finale. Il ne s'agit que de préférences, mais elles correspondent aux positions qu'occupent les particules équivalentes dans les L1 respectives. Toujours est-il que la distribution attestée ché les deux groupes d'apprenants est en parfait accord avec les règles de la LC.

Là où on voit une différence nette par rapport aux productions des francophones, c'est que le's apprenants même avancés semblent n'avoir pas encore acquis l'emploi du pronom contrastif dans ce contexte informationnel : en effet, 
dans chacun des groupes, il n'y a qu'un seul apprenant pour expliciter le DDA de la particule au moyen de ce pronom. Voici l'exemple du polonophone:

(33) il a acheté une échelle

lui aussi veut libérer la princesse (Anna, pol $>$ fr.)

Tout comme chè les débutants - intermédiaires, d'autres moyens sont employés pour le marquage de cette relation, en particulier la comparaison explicite entre les actions des deux personnages au moyen de comme ou de même chose que. Ces moyens restent tout de même réduits par rapport à ceux employés par les locuteurs adultes natifs (cf. tableau 10).

\subsection{Addition d'intervalles temporels}

\subsubsection{Le niveau débutant - intermédiaire}

En ce qui concerne l'addition de procès ou d'intervalles temporels, on peut voir que les apprenants débutants semblent ne pas disposer de moyens spécifiques pour marquer la réitération ou la continuité. Dans ces contextes, ils recourent à l'indication horaire, ou encore ils constatent, tout comme les enfants de 4 ans, la similarité entre les images consécutives pour la continuité:

ici Monsieur beaucoup fatigué

et $\varnothing$ un petit peu dormi

voilà l'église et 3 heures et demie

ici 4 h Monsicur dormi

à 4 h 30 Monsieur regarder le journal

a 5 h monsieur dormi (Wal.)

(35) M. Rouge se couche (=s'allonge ?) devant l'église ici il se couche (= s'allonge ?) aussi (Mat.)

I es intermédiaires utilisent dans les deux cas encore". Ils se démarquent ainsi des natifs francophones qui semblent préférer les moyens spécifiques tels que re-V.

Le placement syntaxique des particules à valeur temporelle est encore incertain. Sur un total de 17 occurrences de encore à valeur temporelle (itérative aussi bien que continuative), on constate 10 occurrences où ces particules se trouvent aprés le verbe fini, et 7 occurrences où elles sont placées soit entre le SN sujet et le verbe, soit en tête d'énoncé, un même apprenant pouvant réaliser alternativement ces différentes positions ${ }^{11}$ :

(36) (a) MR dort à côté de l'église / / encore dort MR lit le journal / / il dort encore (Ren.)

(b) il est toujours à côté de l'église il dort // il toujours dort (Barb.)

9. Un seul sujet utilise toujours pour la continuité.

10. Lees données ne sont malheureusement pas asse\% copieuses pour étayer l'hypothèse yu'à cette différence de position correspondent différentes valeurs sémantiques. 
(c) il dort encore // MR il a reveil ensuite il MR encore il dort (Edyta)

L'observation du marquage explicite des relations temporelles (tous moyens confondus : indication d'horaire, durée ou particule) révèle que, contrairement aux productions enfantines, c'est la continuité qui est signalée par $100 \%$ des sujets, alors que la réitération n'est signalée que par $60 \%$ des sujets. L'explication pourrait résider dans les connaissances discursives de l'apprenant adulte. En effet, contrairement à l'épisode II (continuité), où l'absence d'un marquage spécifique (il dort/il dort) ne permet pas de distinguer la répétition métalinguistique du même énoncé de la continuation de la même action de la part du protagoniste, le non-marquage de la réitération dans la scène III (réitération) n'engendre pas le même doute, puisque les deux occurrences de la même. situation sont séparées par une action différente (lire le journal).

\subsubsection{Le niveau avancé}

L'addition d'intervalles temporels est exprimée de manière similaire par les deux groupes d'apprenants.

$C^{\prime}$ est encore qui figure dans les deux contextes temporels : chez les germanophones, il est employé 6 fois pour la continuation et 4 fois pour la réitération de la même situation (voire même encore une fois chez les plus avancés). On trouve deux occurrences d'encore pour chaque valeur chez les polonophones. Le placement syntaxique de chaque occurrence est systématiquement postverbal, en accord avec la LC.

(37) (a) il dort // il dort encore // il lit le journal // et il dort encore (Seb)

(b) il est endormi sur le banc // il est $12 \mathrm{~h} 20$ et il dort encore // après il s'endort encore une fois (Ilka)

Parmi les autres moyens utilisés figurent les indications horaires, l'expression de la durée et l'emploi ponctuel de aussi. Un seul sujet par groupe utilise toujours, le sujet polonophone l'employant d'ailleurs dans les deux contextes temporels :

(38) il dort dans sa banc $/ /$ il toujours dort $/ /$ il lit un journal // il dort toujours (Marta)

Une différence nette par rapport aux production des francophones concerne l'emploi du moyen spécifique re-V, qui n'est jamais attesté pour la réitération, le seul verbe présentant ce préfixe est « revenir », qui a une signification non pas répétitive mais restitutive.

La simultanéité ne semble pas être exprimée : on remarque l'emploi par deux sujets germanophones et un sujet polonophone de constructions gérondives (il s'arrête avant lé café e'n fumant une cigaretté, Marc; én montant la colline il voit MR, Laurin; il sort en tenant cette échelle, Magda), qui traduisent deux actions simultanées, effectuées cependant par le même sujet. 


\subsection{Résumé 12 : traits développementaux partagés par les adultes}

On a vu, dans les productions adultes, que les relations additives s'établissent dès le début à partir du contexte discursif. Pour la référence aux personnages, on n'observe pas de cas ambigus. La particule aussi constitue le moyen privilégié pour signaler l'addition d'entités. Son DDA lui est adjacent au début; cette particule est placée à partir du niveau intermédiaire après le verbe fléchi (forme linguistique absente des productions des débutants). D'autres travaux (Dimroth 2002, Perdue, Benazzo et Giuliano 2002, Benazzo 2003) analysent de façon plus détaillée l'interaction étroite entre le placement des particules et le début du marquage de la finitude chez les apprenants adultes. Pour ce qui est de l'addition des procès et des intervalles temporels, on constate au début une absence de marquage spécifique, dû sans doute au problème qu'ont ces apprenants à exprimer un procès au moyen du verbe et de ses arguments (Benazzo 2002). A partir du niveau intermédiaire, la particule encore est utilisée pour exprimer tant la réitération que la continuité.

Au niveau intermédiaire, les apprenants adultes disposent donc d'un répertoire basique de moyens lexicaux - essentiellement aussi et encore, mais avec des occurrences de comme $S N$ - leur permettant d'exprimer les relations additives étudiées ici.

Quant aux apprenants avancés, la situation ne change guère (tout au plus remarque-t-on quelques tentatives pour exprimer la simultanéité). Le répertoire acquis de particules est fonctionnel, ce qui semble retarder l'acquisition de moyens plus spécifiques.

Ce que l'on n'observe pas chez ces apprenants est donc l'utilisation de moyens plus "spécifiques " du français LC - le préfixe re-V, le pronom contrastif lui - qui sont très productifs che\% les jeunes Français, ni bien sûr de procédures syntaxiques complexes (subordination) permettant de comparer et de contraster les protagonistes et les actions qu'ils accomplissent.

Cécontraste est visualisé dans le tableau 11 en annexe III.

\section{DISCUSSION : COMPARAISON L1/L2}

Nous avons analysé les productions d'apprenants, enfants et adultes, à qui nous avons demandé de construire un récit à partir d'une série d'images. Par rapport à l'expression des relations additives, nous avons observé une progression similaire chez les enfants francophones, germanophones et polonophones (cf. section 3.3) : ces relations s'établissent d'abord à partir de la situation extralinguistique et ensuite seulement à partir du contexte discursif. On rejoint ici, par un autre biais, une observation déjà ancienne de Hickmann (1982) sur le développement de la compétence méta-pragmatique chez l'enfant : les enfants 
de quatre ans éprouvent des difficultés à employer le langage comme contexte pour le langage. Nous avons également observé que ce n'est qu'à dix ans que les enfants (dans la tâche analysée du moins) sortent de la contrainte de l'ordre chronologique, elle-même basée sur l'extra-linguistique. Troisième observation, le sur-marquage de certaines relations additives chez les enfants de sept ans, ce qui témoigne de l'ajustement de formes linguistiques, présentes dans le répertoire enfantin, aux contextes discursifs appropriés. Cette observation rejoint les résultats de Hendriks (2000) et d'autres sur le maintien de la référence personnelle. Nous avons observé, enfin, un développement dans l'expression des relations additives elles-mêmes qui affine celui proposé par hypothèse dans l'introduction, et répété ici :

(fonction de) alussi $>$ (fonction de) encore $_{1,2}>$ (fonction de) encore ${ }_{3}$, toujours

Le développement des particules employées manifeste une transition entre :

addition d'entités (extradiscursives > intradiscursives) : aussi (et équivalents)

$>$ addition d'occurrences de procès : encore 2 (et équivalents)

(construction d'une chronologie narrative)

$>$ addition d'intervalles temporels encore ${ }_{3}$, toujours (et équivalents)

$>$ même intervalle temporel pour 2 situations

(subordination plutôt que l'emploi des particules comme moyen de contraster deux personnages/actions)

$C^{\prime}$ est au départ de cette séquence que nous avons observé, dans les trois langues étudiées, des utilisations inappropriées de certaines particules. Ce développement dans l'expression des relations additives reflète le développement global de la capacité discursive chez l'enfant.

Nous avons observé dans les productions d'apprenants adultes également un développement dans la nature des relations additives exprimées qui reflète celui esquissé ci-dessus, ainsi que celui analysé par Benazzo (2002) :

aussi (et équivalents) $>$ encore $_{2,3}$ (et équivalents) $>$ (simultanéité)

mais qu'on ne peut expliquer de la même façon - en effet, les adultes s'exprimant dans leur langue maternelle utilisent l'ensemble des relations additives. $C^{\prime}$ est ici qu'on voit clairement la différence de nature des facteurs déterminant deux progressions montrant des similarités superficielles: la capacité expressive du répertoire linguistique du moment détermine l'ordre d'emploi des particules chez les adultes, alors que c'est l'apprentissage progressif d'opérations discursives qui éclaire le mieux le développènent enfantin.

Pour ce qui est des moyens linguistiques utilisés, nous constatons, une fois le stade initial dépassé, des divergences frappantes. Tandis que des moyens linguistiques divergents et spécifiques se développent entre 7 ans et 10 ans che\% les enfants francophones et polonophones, les enfants germanophones 
(tout comme les adultes, d'ailleurs) continuent d'utiliser presque exclusivement les particules additives, comme l'attestent les tableaux 10 et 11 . Le Tableau 5, qui donne la fréquence globale d'utilisation des particules, nous montre que le corpus germanophone en contient 327 , tandis que le corpus polonophone en contient 133 seulement, et le corpus francophone (qui regroupe le plus de locuteurs) 168. Cette tendance est indirectement confirmée si on compare les productions en L2 des deux groupes avancés 52 occurrences chez les germanophones contre 30 chez. les polonophones "I.

Il s'avère que l'organisation de l'énoncé additif en allemand (pour la narration, du moins) est très régulière et déjà en place à 4 ans. L'analyse des 23 énoncés contenant auch à 4 ans montre que cette particule est systématiquement placée dans le Mittelfeld, derrière le verbe fléchi. Les germanophones continuent d'employer ce schéma, en l'élaborant quelque peu ${ }^{12}$, et se servent très peu d'autres moyens pour exprimer les relations additives. Il est remarquable, par exemple, que les moyens de subordination pour mettre en contraste les personnages et leurs actions se réduisent, chez les adultes germanophones, à quatre occurrences de während (pendant que) produits par seulement deux locuteurs.

La tâche acquisitionnelle des enfants germanophones à partir de 4 ans est donc principalement de distinguer les différentes valeurs de ces particules, car le placement préférentiel dans une position dominante et la grande fréquence de ces particules dans l'input facilite l'acquisition précoce de cette même position. La mobilité syntaxique de aussi et tez se reflète dans les productions des très jeunes enfants francophones et polonophones, mais leur évolution ultérieure dépend de la variation dans l'ordre des mots en polonais et du développement de moyens spécifiques pour la référence aux entités en français. Les locuteurs natifs de ces langues utilisent, comme nous l'avons vu, des moyens plus variés pour exprimer les relations additives et la maîtrise de cet aspect de la construction du discours, au-delà de l'utilisation précoce des particules, demande plus de temps.

Il n'en reste pas moins que ces particules sont "centrales " dans l'ensemble des parcours acquisitionnels que nous avons suivis dans cet article. Ce sont des lexèmes saillants, invariables, qui représentent pour l'ensemble des apprenants le point de départ de l'expression des relations additives, et pour les apprenants adultes, sans doute, le point d'arrivée... aussi.

11. Il s'agit done non pas d'un transfert simple d'un moyen linguistique, mais du transfert du poids d'un moyen linguistique dans la construction du discours.

12. Les apprenants germanophones du français adoptent massivement (Tableau 9) le schéma Équivalent $\mathrm{SN}+\mathrm{V}$ fléchi + aussi $+\mathrm{X}$. 


\section{Références}

BECKER, A. \& R. DIETRICH (1996). The acquisition of scope in L2 German. Zeitschrift für Litteraturwissenschaft und Linguistik 104, 115-40.

BENAZZO S. (2002). Communicative potential vs structural constraints: explanatory factors for the acquisition of scope particles, Eurosla Yearbook 2, Amsterdam, Benjamins, 187204.

BENAZZO S. (2003). The interaction between verb morphology and temporal adverbs of contrast. A longitudinal study in French, English and German L2. In C. Dimroth et M. Starren (éds.) Information structure, linguistic structure and the dynamics of language acquisition, Amsterdam, Benjamins, 187-210.

DIMROTH, Ch. (1998). Fokuspartikeln und Informationgliederung im Diskurs. Thèse. Freie Universität Berlin.

DIMROTH, Ch. (2002). Topics, assertions, and additive words: how L2 learners get from information structure to target-language syntax. Linguistics 40 (4), 891-923.

DIMROTH, C. \& W. KLEIN (1996). Fokuspartikeln in Lernervarietäten. Zeitschrift für Litteraturwissenschaft und Linguistik 104, 73-114.

GayraUd, F. (2004). Émergence et développement du placement des particules de portée, Acquisition et Interaction en Langue Étrangère 20, 173-196.

HENDRIKS, H. (2000). The acquisition of topic marking in L1 Chinese and L1 and L2 French. Studies in Second Language Acquisition 22, 369-397.

HICKMANN, M. (1982). The development of narrative skills. Pragmatic and metapragmatic aspects of discourse cohesion. Unpublished doctoral dissertation. University of Chicago.

JOHNSON, J. \& D. SLOBIN (1979). The development of locative expressions in English, Italian, Serbo-Croatian and Turkish. Journal of Child Language 6, 529-45.

KöNIG, E. (1991). The meaning of focus particles. London : Routledge.

KRIFKA, M. (1998). Additive particles under stress. Ms. Berlin.

LASSER, I. (1997). Finiteness in adult and child German. MPI Series in Psycholinguistics. Nijmegen : Max-Planck-Institut.

Nederstigt U. (2004). Auch and noch in child and adult German. Berlin, New York: de Gruyter.

Perdue, C., S. Benazzo \& P. Giuliano (2002). When finiteness gets marked. Linguistics 40 (4), 849-90.

REIS, M. \& I. ROSENGREN (1997). A modular approach to the grammar of additive particles: the case of German auch. Journal of Semantics 14 (4).

SLOBIN, D. (1978). A case study of early language awareness. In A. Sinclair, R. Jarvella \& W. Levelt (Dir), The child's conception of language, Language, Berlin : Springer, 45-54. 
ANNEXE 1: Addition d'entités en L1

Tableau 6.1 : Moyens utilisés pour marquer l'addition d'entités en français

\begin{tabular}{|l|l|c|c|c|c|}
\hline & & $\mathbf{4}$ ans & $\mathbf{7}$ ans & $\mathbf{1 0}$ ans & Adultes \\
\hline Aussi & & $\mathbf{1 4}$ & $\mathbf{1 1}$ & $\mathbf{9}$ & $\mathbf{9}$ \\
\hline \hline \multirow{2}{*}{ autres moyens } & comme $X$ & - & 2 & 2 & 4 \\
\cline { 2 - 6 } & À son tour & - & - & - & 2 \\
\cline { 2 - 6 } & également & - & - & - & 5 \\
\cline { 2 - 6 } & même $+\mathrm{N}$ & $(1)$ & - & 1 & 2 \\
\hline \hline Total & & $\mathbf{1 5}$ & $\mathbf{1 3}$ & $\mathbf{1 2}$ & $\mathbf{2 2}$ \\
\hline \hline
\end{tabular}

Tableau 6.2 : Moyens utilisés pour marquer l'addition d'entités en allemand (uniquement dans les épisodes selectionnés)

\begin{tabular}{|l|l|c|c|c|c|}
\hline & & $\mathbf{4}$ ans & $\mathbf{7}$ ans & $\mathbf{1 0}$ ans & Adultes \\
\hline auch & & $\mathbf{8}$ & $\mathbf{1 8}$ & $\mathbf{2 4}$ & $\mathbf{2 5}$ \\
\hline \hline \multirow{3}{*}{ autres moyens } & wieder & $14^{*}$ & - & - & - \\
\cline { 2 - 6 } & ebenfalls & - & - & - & 2 \\
\cline { 2 - 6 } & wie X & - & 1 & - & 2 \\
\hline Total & & $\mathbf{2 2}$ & $\mathbf{1 9}$ & $\mathbf{2 4}$ & $\mathbf{2 9}$ \\
\hline
\end{tabular}

* inappropricici, of ex. 10.

Tableau 6.3 : Moyens utilisés pour marquer l'addition d'entités en polonais

\begin{tabular}{|l|l|c|c|c|c|}
\hline & & $\mathbf{4}$ ans & $\mathbf{7}$ ans & $\mathbf{1 0}$ ans & Adultes \\
\hline \multirow{2}{*}{ tez } & & $\mathbf{9}$ & $\mathbf{1 5}$ & $\mathbf{1 3}$ & $\mathbf{2 1}$ \\
\hline \hline \multirow{4}{*}{ autres moyens } & takze/ rowniez & - & - & - & 15 \\
\cline { 2 - 6 } & tak samo (jak) & - & 2 & 1 & - \\
\cline { 2 - 6 } & podobnie jak X & - & 2 & - & 4 \\
\cline { 2 - 6 } & to samo./sam + N & - & 1 & - & 3 \\
\hline \hline tot. & & $\mathbf{9}$ & $\mathbf{2 0}$ & $\mathbf{1 4}$ & $\mathbf{4 3}$ \\
\hline \hline
\end{tabular}


Tableau 7.1 : Positions de aussi dans des structures à verbe explicite

\begin{tabular}{|c|c|c|c|c|c|}
\hline & & 4 ans & 7 ans & 10 ans & Adultes \\
\hline initiale & initiale (aussi SN V) & 1 & - & - & - \\
\hline \multirow[t]{2}{*}{ préverbale } & $\begin{array}{l}\text { après le } S N \text { sujet } \\
(\mathrm{SN} \text { aussi } \mathrm{V} . . .)\end{array}$ & 1 & 1 & - & 1 \\
\hline & $\begin{array}{l}\text { Iui aussi en tête d'énoncé } \\
\text { (lui aussi }+ \text { pro } \vee . . . \text { ) }\end{array}$ & - & 4 & 1 & 4 \\
\hline \multirow[t]{3}{*}{ postverbale } & $\begin{array}{l}\text { lui aussi en pos. postverbale } \\
\text { (SN (Aux)-V lui aussi...) }\end{array}$ & - & 1 & 2 & 1 \\
\hline & $\begin{array}{l}\text { postverbale } \\
(\mathrm{SN}+(\text { Aux })-\mathrm{V} \text { aussi) }\end{array}$ & 5 & 2 & 5 & 1 \\
\hline & $(+V$ aussi... & - & - & - & 1 \\
\hline finale & $\begin{array}{l}\text { après l'objet } \\
\text { SN (Aux)-V SN/SP aussi }\end{array}$ & 3 & 1 & - & - \\
\hline \multirow[t]{2}{*}{ autres } & & 1 & - & - & 1 \\
\hline & tot. occ. & $\overline{11}$ & 9 & 8 & 9 \\
\hline
\end{tabular}

Tableau 7.2 : Positions de auch dans des structures à verbe explicite

\begin{tabular}{|l|l|r|r|r|c|}
\hline & & $\mathbf{4}$ ans & $\mathbf{7}$ ans & $\mathbf{1 0}$ ans & Adultes \\
\hline \hline initiale & initiale (auch SN V) & - & - & - & 2 \\
\hline \hline \multirow{3}{*}{ Mittelfeld } & SN/pro/Ø Vfin auch & $\mathbf{1 2}$ & $\mathbf{1 4}$ & $\mathbf{1 9}$ & $\mathbf{1 3}^{\star}$ \\
\cline { 2 - 6 } & (adv) Vfin SN/pro auch & 10 & 7 & 5 & 5 \\
\cline { 2 - 6 } & adv Vfin auch SN & - & 2 & 2 & 2 \\
\hline \hline autres & & - & - & - & - \\
\hline \hline & total occ. & 22 & 23 & 26 & 22 \\
\hline
\end{tabular}

*dont 2 fois ebenfalls

Tableau 7.3 : Positions de tez dans des structures à verbe explicite

\begin{tabular}{|l|l|c|c|c|c|}
\hline & & $\mathbf{4}$ ans & $\mathbf{7}$ ans & $\mathbf{1 0}$ ans & Adultes \\
\hline \hline \multirow{2}{*}{ initiale } & tez V & $\mathbf{6}$ & $\mathbf{6}$ & $\mathbf{4}$ & $\mathbf{1 0}$ \\
\cline { 2 - 6 } & tez SN V & - & - & 1 & 3 \\
\hline \hline après le sujet & SN tez V... & 1 & 6 & 7 & 3 \\
\hline \hline \multirow{2}{*}{ après le V } & SN / V tez. & 1 & 1 & 2 & 4 \\
\cline { 2 - 6 } & V tez SN & - & - & - & 2 \\
\hline \hline \multirow{2}{*}{ finale } & $\begin{array}{l}\text { (après le complément du } \\
\text { verbe) }\end{array}$ & - & - & - & - \\
\hline \hline & total occ. & 8 & 13 & 14 & 22 \\
\hline
\end{tabular}


ANNEXE II : Addition d'intervalles temporels en L1

Tableau 8.1 : Addition temporelle en français L1

\begin{tabular}{|c|c|c|c|c|c|}
\hline & item & $\begin{array}{c}4 \text { ans } \\
\text { (15 suj.) }\end{array}$ & $\begin{array}{c}7 \text { ans } \\
\text { (15 suj.) }\end{array}$ & $\begin{array}{c}10 \text { ans } \\
\text { (15 suj.) }\end{array}$ & $\begin{array}{l}\text { Adultes } \\
\text { (10 suj.) }\end{array}$ \\
\hline \multirow[t]{3}{*}{ Réitération } & encore & 5 & 2 & - & - \\
\hline & $\mathbf{R e}-\mathbf{V}$ & 5 & 8 & 13 & 7 \\
\hline & autre & - & $\begin{array}{c}1 \\
\text { (continuer*) }\end{array}$ & $\begin{array}{c}1 \\
(H)\end{array}$ & $\begin{array}{c}2 \\
(H+\text { de nou- } \\
\text { veau })\end{array}$ \\
\hline \multirow[t]{3}{*}{ Continuitē } & encore & 6 & 5 & 6 & 1 \\
\hline & toujours & - & 4 & 6 & 8 \\
\hline & autre & $\begin{array}{c}2 \\
\text { (aussi }+ \\
\text { longtps) }\end{array}$ & $\begin{array}{c}1 \\
\text { (continuer) }\end{array}$ & $\begin{array}{c}3 \\
\text { (continuer, } \mathrm{H}, \\
\text { longtemps) }\end{array}$ & $\begin{array}{c}1 \\
(H)\end{array}$ \\
\hline \multirow[t]{2}{*}{ Simultanéité } & pendant & - & 1 & 5 & 2 \\
\hline & autre & - & - & - & 3 \\
\hline
\end{tabular}

*cotte utilisation de contimaer est imappropries par rapport a la production adulte

II heure' : signifie que le sujet fait appel a l'heure sur l'horloge pour exprimer ces valeurs.

Tableau 8.2 : Addition temporelle en allemand L1

\begin{tabular}{|c|c|c|c|c|c|}
\hline & item & $\begin{array}{c}4 \text { ans } \\
\text { (10 suj.) }\end{array}$ & $\begin{array}{l}7 \text { ans } \\
\text { (8 suj.) }\end{array}$ & $\begin{array}{l}10 \text { ans } \\
19 \text { suj.) }\end{array}$ & $\begin{array}{l}\text { Adultes } \\
\text { (9 suj.) }\end{array}$ \\
\hline \multirow[t]{2}{*}{ Réitération } & (schon) wieder & 4 & $8(=100 \%)$ & $9(=100 \%)$ & 6 \\
\hline & autre & - & - & - & 2 \\
\hline \multirow[t]{3}{*}{ Continuité } & (schon) wieder & $4 *$ & $1 *$ & $1^{*}$ & - \\
\hline & immer noch & 3 & 5 & 4 & 7 \\
\hline & autre & $2\left(\right.$ auch $\left.^{*}\right)$ & 1 (weiter) & 3 & 1 (weiter) \\
\hline \multirow[t]{2}{*}{ Simultanéité } & während & - & - & - & 4 \\
\hline & autre & - & - & - & - \\
\hline
\end{tabular}

*anch et *uieder utilisés en tant qu'addition déictique, et de façon inappropriée à 4 ans.

A 7 ans le contraste entre ádeder (réiteration) et immer noh (continuité) est prespue systématicuement marqué. Ie marquage de la simultanéité che\% les adultes est limité à 4 oce. produites par 2 sujets seulement. 
Tableau 8.3 : Addition temporelle en polonais L1

\begin{tabular}{|c|c|c|c|c|c|}
\hline & item & $\begin{array}{c}4 \text { ans } \\
\text { (10 suj.) }\end{array}$ & $\begin{array}{c}7 \text { ans } \\
(10 \text { suj. })\end{array}$ & $\begin{array}{l}10 \text { ans } \\
(10 \text { suj.) }\end{array}$ & $\begin{array}{l}\text { Adultes } \\
\text { (10 suj.) }\end{array}$ \\
\hline \multirow[t]{3}{*}{ Réitération } & jeszcze (encore) & - & - & - & - \\
\hline & $\begin{array}{l}\text { znowu } \\
\text { (de nouveau) }\end{array}$ & 2 & 8 & 9 & 4 \\
\hline & autre & $2($ tez $*)$ & 1 (dalej*) & - & 3 \\
\hline \multirow[t]{3}{*}{ Continuité } & jeszcze (encore) & - & - & 2 & 1 \\
\hline & $\begin{array}{l}\text { nadal, wciaz, dalej } \\
\text { (toujours) }\end{array}$ & 1 & 3 & 1 & 4 \\
\hline & autre & - & $\begin{array}{c}2 \\
(\mathrm{H}+\text { znowu* }) \\
\end{array}$ & $\begin{array}{c}2 \\
\left(\operatorname{tez} z^{*}\right) \\
\end{array}$ & $\begin{array}{c}4 \\
(H)\end{array}$ \\
\hline Simultanéité & $\begin{array}{l}\text { gdy, tymczasem } \\
\text { w tym czasie } \\
\text { (pendant que/ } \\
\text { entretemps) }\end{array}$ & - & - & 3 & 13 \\
\hline
\end{tabular}

*ces utilisations sont inappropriées.

ANNEXE III : Addition en L2

Tableau 9 : Position de aussi dans des structures à verbe explicite en français L2

\begin{tabular}{|c|c|c|c|c|}
\hline & & déb. et interm. & avancés pol. & avancés germ. \\
\hline Initiale & initiale & 2 & - & - \\
\hline \multirow[t]{2}{*}{ après le sujet } & $\begin{array}{l}\text { après le SN sujet } \\
\text { (SN + aussi) }\end{array}$ & 2 & 4 & 1 \\
\hline & $\begin{array}{l}\text { initiale } \\
\text { (lui + aussi) }\end{array}$ & - & 1 & 2 \\
\hline \multirow[t]{2}{*}{ après le V } & $\begin{array}{l}\text { postverbale } \\
\mathrm{SN}+(\text { Aux }) \vee \text { lui aussi... }\end{array}$ & - & - & - \\
\hline & $\begin{array}{l}\text { postverbale } \\
S N+V \text { aussi }(x x)\end{array}$ & 12 & 10 & 26 \\
\hline Finale & $\begin{array}{l}\text { finale } \\
S N+(A u x) V+A N / S P \text { aussi }\end{array}$ & - & 1 & 4 \\
\hline \multirow[t]{2}{*}{ Autres } & & 2 & 1 & 1 \\
\hline & tot. occ. & 18 & 17 & 34 \\
\hline
\end{tabular}


Tableau 10 : Moyens utilisés pour marquer l'addition d'entités en français L2

\begin{tabular}{|l|l|c|c|c|c|}
\hline & & $\begin{array}{c}\text { déb. et } \\
\text { interméd. }\end{array}$ & avancés pol. & $\begin{array}{c}\text { avancés } \\
\text { germ. }\end{array}$ & $\begin{array}{c}\text { adultes } \\
\text { franc. natifs }\end{array}$ \\
\hline Aussi & & $\mathbf{1 8}$ & $\mathbf{1 7}$ & $\mathbf{3 4}$ & $\mathbf{9}$ \\
\hline Autres moyens & & $\mathbf{6}$ & $\mathbf{4}$ & $\mathbf{3}$ & $\mathbf{1 3}$ \\
\hline \hline \multirow{3}{*}{ Dont } & comme $X$ & 3 & 3 & 2 & 4 \\
\cline { 2 - 6 } & à son tour & - & - & - & 2 \\
\cline { 2 - 6 } & également & - & - & - & 5 \\
\cline { 2 - 6 } & méme N & 2 & 1 & - & 2 \\
\cline { 2 - 6 } & autres & 1(à nouveau) & - & 1 (encore) & - \\
\hline
\end{tabular}

Tableau 11 : Itération et continuation chez les apprenants L2

\begin{tabular}{|c|c|c|c|c|c|}
\hline & item & $\begin{array}{l}\text { déb./interm. } \\
\text { (10 sujets| }\end{array}$ & $\begin{array}{c}\text { avancés pol. } \\
\text { (10 suj.) }\end{array}$ & $\begin{array}{c}\text { avancés germ. } \\
\text { (10 suj.) }\end{array}$ & $\begin{array}{l}\text { Adultes natifs } \\
\qquad(10 \text { suj.) }\end{array}$ \\
\hline \multirow[t]{3}{*}{ Réitération } & encore & 4 & 2 & 4 & - \\
\hline & $\mathrm{Re} \cdot \mathrm{V}$ & - & - & - & 7 \\
\hline & autre & 2 (heure) & $\begin{array}{l}2 \text { (heure }+ \\
\text { toujours }{ }^{*} \text { ) }\end{array}$ & $\begin{array}{l}5 \text { (heure + } \\
\text { aussi) }\end{array}$ & 2 \\
\hline \multirow[t]{3}{*}{ Continuité } & encore & 5 & 2 & 6 & 1 \\
\hline & toujours & 1 & 1 & 1 & 8 \\
\hline & autre & $4(H+$ aussi $)$ & $5(h+$ aussi $)$ & $2(H+V)$ & 1 \\
\hline \multirow[t]{2}{*}{ Simultanéité } & pendant & - & - & - & 2 \\
\hline & autres & - & - & - & 3 \\
\hline
\end{tabular}

* foufours inapproprie, voir l'exemple (38) 